\title{
qMrdd2, a novel quantitative resistance locus for maize rough dwarf disease
}

\author{
Weixiao Zhang ${ }^{1}$, Suining Deng ${ }^{2}$, Yan Zhao ${ }^{1}$, Wei Xu' ${ }^{1}$, Qingcai Liu² ${ }^{2}$ Yongzhong Zhang ${ }^{1}$, Chunmei Ren ${ }^{3}$, \\ Zhaobang Cheng ${ }^{3}$, Mingliang $\mathrm{Xu}^{2}$ and Baoshen Liu ${ }^{1 *}$
}

\begin{abstract}
Background: Maize rough dwarf disease (MRDD), a widespread disease caused by four pathogenic viruses, severely reduces maize yield and grain quality. Resistance against MRDD is a complex trait that controlled by many quantitative trait loci (QTL) and easily influenced by environmental conditions. So far, many studies have reported numbers of resistant QTL, however, only one QTL have been cloned, so it is especially important to map and clone more genes that confer resistance to MRDD.

Results: In the study, a major quantitative trait locus (QTL) qMrdd2, which confers resistance to MRDD, was identified and fine mapped. $q$ Mrdd2, located on chromosome 2, was consistently identified in a 15-Mb interval between the simple sequence repeat (SSR) markers D184 and D1600 by using a recombinant inbred line (RIL) population derived from a cross between resistant ("80007") and susceptible ("80044") inbred lines. Using a recombinant-derived progeny test strategy, aMrdd2 was delineated to an interval of $577 \mathrm{~kb}$ flanked by markers N31 and N42. We further demonstrated that $9 M$ rdd2 is an incompletely dominant resistance locus for MRDD that reduced the disease severity index by $20.4 \%$.
\end{abstract}

Conclusions: A major resistance QTL ( $q$ Mrdd2) have been identified and successfully refined into $577 \mathrm{~kb}$ region. This locus will be valuable for improving maize variety resistance to MRDD via marker-assisted selection (MAS).

Keywords: Maize, MRDD, QTL, Fine-mapping, MAS

\section{Background}

Maize rough dwarf disease (MRDD) is a serious viral disease worldwide. MRDD is caused by four pathogenic viruses [1], namely, Maize rough dwarf virus (MRDV), Mal de Rio Cuarto virus (MRCV), Rice black-streaked dwarf virus (RBSDV) and Southern rice black-streaked dwarf virus (SRBSDV), which are prevalent in Europe [2], South America [3], Asia [4], and Northern China [5], respectively. These pathogenic viruses are naturally transmitted by the small brown planthopper Laodelphax striatellus in a persistent manner [6] and are not spread

\footnotetext{
*Correspondence: liubs@sdau.edu.cn

1 State Key Laboratory of Crop Biology, Shandong Agricultural University,

61 Daizong Street, Taian 271018, People's Republic of China

Full list of author information is available at the end of the article
}

by seed or mechanical inoculation. Once infected, L. striatellus can transmit these viruses for life, though transmission to eggs does not occur [7].

MRDD causes substantial yield losses in maize production globally [2]. Indeed, between 2008 and 2011, MRDD was responsible for yield losses among more than 3,000,000 ha of crops each year in North China with yield losses reaching $100 \%$ in some seriously affected areas [8]. The disease leads to severe symptoms, such as plant dwarfing, internode shortening, waxy enation, malformed tassels and dark-green leaves [9]. Current methods for controlling MRDD are agricultural measures (i.e., delayed sowing) and chemical control. However, delayed sowing leads to the underutilization of photothermal resources and renders the seedlings vulnerable to waterlogging and late ripening, 
as occurred in the Yellow-Huai-Hai River plain [10]. Small-scale chemical control is also not ideal and leads to environmental pollution. Accordingly, cloning natural resistance genes and breeding resistant maize varieties would be the most environmentally responsible and cost-effective means of controlling MRDD. Hence, collecting and identifying various resistant germplasms will facilitate the development of resistant maize cultivars, and it is especially important to map and clone resistant genes to MRDD.

Most research indicates that maize resistance to MRDD is controlled by many genes, each with different effects $[2,11,12]$. Maize germplasms shows different levels of resistance to MRDD under natural conditions, with the most resistant germplasm originating from US hybrid P78599 [13-16]. By using $\mathrm{F}_{3}$ families of Mo17 and BSL14, Di Renzo et al. [17] found that in Argentina, the heritability of MRCV resistance in maize ranged from 0.44 to 0.56 , and based on an $\mathrm{F}_{2: 3}$ QTL-mapping strategy, 2 quantitative trait loci (QTLs) were localized to maize chromosome bins 1.03 and 8.03/4 [18]. Luan et al. [19] screened four linked molecular markers and mapped three QTLs for resistance to MRDD within chromosome bins $6.02,7.02$ and 8.07 by using an $F_{2}$ population derived from the resistant inbred line 90,110 and the susceptible inbred line Ye478. Through association analysis with 163 inbred lines, Shi detected one associated single nucleotide polymorphism (SNP) in the ZmeIF4E promoter, which accounting for 3.33 and $9.04 \%$ of the phenotypic variation, respectively in two environments, [20]. Using 152 maize germplasm isolates and 89 recombinant inbred lines (RILs) derived from resistant line X178 and susceptible line B73, Shi et al. [21] detected three QTLs for MRDD resistance in bins 2.07, 5.04 and 8.03, and the QTL on bin 8.03 explained $24.6-37.3 \%$ of the phenotypic variance. This major QTL ( $q M r d d 8)$ on chromosome bin 8.03 was then fine-mapped to a $347-\mathrm{kb}$ region by Shi's laboratory [22]. With 527 inbred lines and 556,000 SNPs, 15 candidate genes associated with MRDD resistance have been identified [23]. Conventional linkage and high-throughput sequencing analysis have been employed to locate the resistance-related genomic region, and an associated region was identified within the genomic interval $68,396,487-69,523,478$ bp on chromosome 6 [24]. A major QTL ( $q M r d d 1)$ that reduces the DSI by $24.2-39.3 \%$ was fine-mapped to a $1.2-\mathrm{Mb}$ region by applying recombinant-derived progeny testing to selfpollinated backcrossed families [25]. Although many QTLs have been identified, few have been fine-mapped or even cloned. Liu et al. (2020) reported that the Rab GDP dissociation inhibitor $(Z m G D I \alpha)$ is a candidate gene for $q M r d d 1$ which confers recessive resistance to MRDD and resolves the underlying molecular mechanisms controlling viral diseases. $q M r d d 1$ is also the only resistance gene cloned to date [26].

Considering the damage caused by MRDD and the complexity of disease resistance, it is critical to identify new resistance QTLs that can be used to genetically control MRDD and to deepen our understanding of resistance mechanisms.

\section{Results}

\section{Initial QTL analysis of MRDD}

For each field trial, the RIL population showed continuous variation in DSI (range 0-100\%), with averages of 54.2 and $55.5 \%$ in two field trials (2013-A and 2013-B) respectively (Fig. 1a). Spearman's rank correlation coefficient $\left(r_{s}\right)$ among two sets of phenotypic data was calculated. The data among two replications were significantly correlated $\left(\mathrm{p}\right.$-value $=6.74 \mathrm{E}^{-13}$ ) with $r_{s}$ was 0.49 . The broad-sense heritability $\left(H^{2}\right)$ of disease resistance was estimated to be 0.66 (Table S1), and the variance was mainly derived from differences among RILs (Table 1). These results suggested that the genetic factor plays a major role in maize resistance to MRDD within the RIL population.

Two maize lines, "80007" and "80044", were evaluated for resistance to MRDD under artificial inoculation 3 times: in 2017 and 2019 in Taian and in 2018 in Hainan. For each field trial, line "80007" showed effective resistance to MRDD, whereas line "80044" was highly susceptible; the average DSIs of "80007" and "80044" were 34.6 and $82.03 \%$, respectively (Fig. 1b, c).

\section{Initial QTL mapping of MRDD resistance}

Based on 199 RILs and 804 informative SNPs, we constructed a genetic linkage map that covered $1291.05 \mathrm{cM}$, with an average of $1.61 \mathrm{cM}$ between adjacent markers (Fig. S1, Fig. S2, Table S2 and Table S3). The DSI for the 199 RILs was used as phenotypic data for QTL mapping of MRDD resistance. Two QTLs were consistently detected in all three field trials (2013-A, 2013-B and the average DSI phenotype of each RILs in 2013-A and 2013-B) (Fig. S3, Fig. S4 and Table 2). One of the QTLs, $q M r d d 2$, is located on chromosome 2 within a $15-\mathrm{Mb}$ region and explained $12.88-15.67 \%$ of the total phenotypic variation. Another QTL, located on chromosome 5, explained $\sim 6 \%$ of the total phenotypic variation (Table 2 ).

\section{Fine mapping of $q M \operatorname{Mrd} 2$}

According to the initial QTL analysis, two flanking markers (D184 and D1600) and one marker residing within the $q M r d d 2$ region (D829-0) were used to screen the RIL population. Ultimately, six recombinant RILs that had crossover breakpoints within the $q M r d d 2$ region were detected and further genotyped with seven newly isolated 


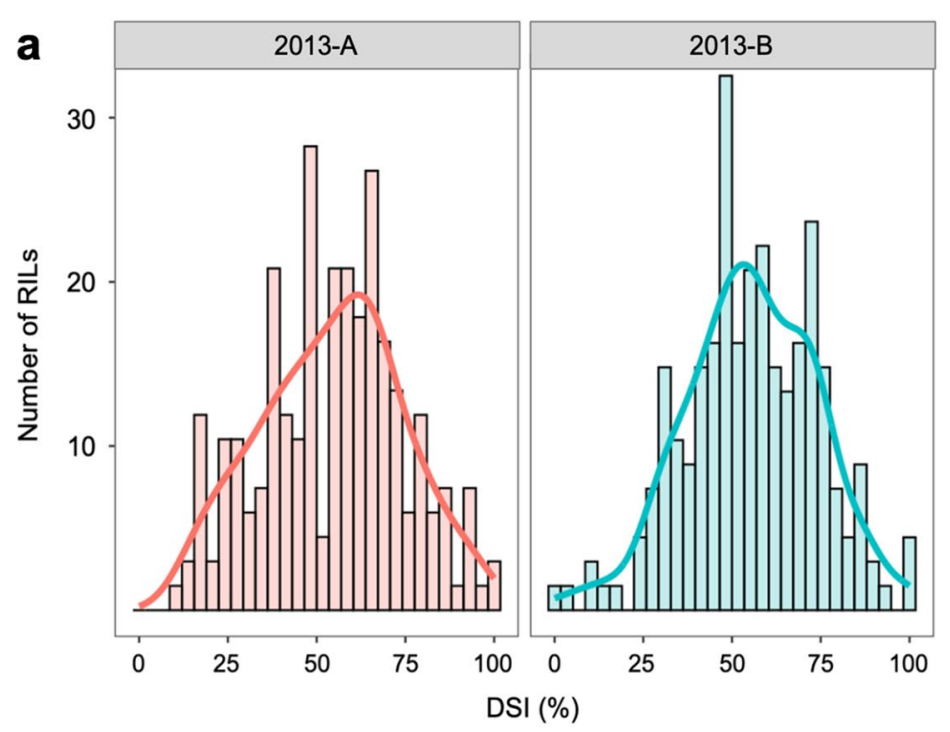

b

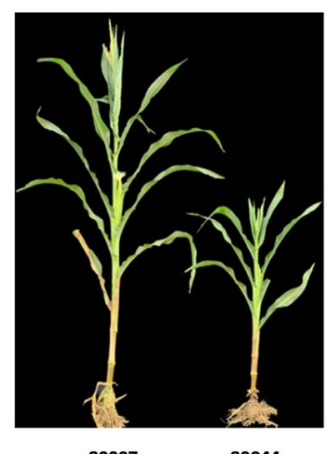

80007
C

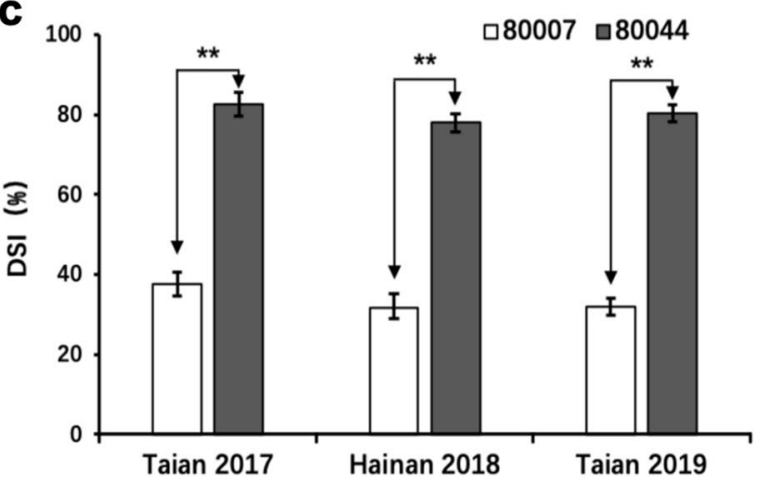

Fig. 1 The phenotype of MRDD in the RILs and parental lines. a Frequency distribution of DSI (\%) in the RIL population in two field trials (2013-A and 2013-B). The number of individuals in each phenotypic class is indicated on the $y$-axis and the phenotypic score classes on the $x$-axis. $\mathbf{b}$ Individual plants from parental lines "80007" and "80044" after artificial inoculation. c The DSI of the parental lines in Taian in 2017 , Hainan in 2018 and Taian in 2019 (**P $<0.01$; two-tailed Student's t-test)

markers (D387-2, D550, D664, D829-0, D936-2, D122-6 and D1347) (Table S4). All six $\mathrm{F}_{5}$ recombinants were self-pollinated to produce corresponding $\mathrm{F}_{6}$ progeny. In the summer of 2017 , the $F_{6}$ progeny (1010 plants) were planted in Taian to test for MRDD resistance in the field. Three genotypes within each $\mathrm{F}_{6}$ progeny derived from individual heterozygous recombinant plants were categorized: qMrdd2 (80007) homozygote and heterozygote and nonqMrdd2 (80044) homozygote. The DSI value for each genotype was calculated. MRDD resistance among the three genotypes of recombinant types a-I, a-III, a-IV, a-V and a-VI was not significantly different $(P<0.05)$, indicating that $q M r d d 2$ is located in the homozygous region of the parental region. For the remaining recombinant type a-II, a statistically significant difference $(P<0.05)$ in MRDD resistance among the three genotypes was observed, suggesting the presence of $q M r d d 2$ in the heterozygous region of the parental recombinant. This analysis shows that the $q M r d d 2$ locus was localized to between

Table 1 Analysis of variance of data in the RIL population

\begin{tabular}{llllrr}
\hline Sources & $\boldsymbol{d f}$ & SS & MS & F-value & Variance \\
\hline RILs & 199 & 65.53 & 0.29 & 8.67 & $3.66 \mathrm{E}-76^{* * *}$ \\
Environments & 2 & 2.17 & 1.13 & 76.0 & $8.23 \mathrm{E}-26 * * *$ \\
Residuals & 398 & 17.3 & $3.16 \mathrm{E}-03$ & $5.16 \mathrm{E}-03$ & $2.15 \mathrm{E}-03$
\end{tabular}

Sources Variation sources, $d f$ Degrees of freedom, SS Sum of squares, MS Mean squares, $P$-value Significant difference among sources

${ }^{* * *} P<0.001$ 
Table 2 The QTL related to MRDD in three field trials

\begin{tabular}{|c|c|c|c|c|c|c|}
\hline Field trial & Chromosome & Position (Mb) & LOD & Additive effect & SRA & $R^{2}$ \\
\hline \multirow[t]{5}{*}{ Average } & 2 & $15.10-39.30$ & 5.5581 & -0.0503 & 80007 & 0.1156 \\
\hline & 3 & $20.42-27.67$ & 3.3725 & -0.0397 & 80007 & 0.0730 \\
\hline & 5 & $50.90-70.26$ & 3.8923 & -0.0446 & 80007 & 0.0625 \\
\hline & 8 & $200.13-219.86$ & 2.8755 & -0.0350 & 80007 & 0.0566 \\
\hline & 10 & $0.44-16.38$ & 3.1656 & -0.0370 & 80007 & 0.0617 \\
\hline \multirow[t]{2}{*}{ R 1} & 2 & $14.80-37.48$ & 5.1172 & -0.0675 & 80007 & 0.1588 \\
\hline & 5 & $54.55-70.26$ & 2.9262 & -0.0425 & 80007 & 0.0614 \\
\hline \multirow[t]{3}{*}{ R 2} & 2 & $13.41-37.48$ & 4.8036 & -0.0648 & 80007 & 0.1288 \\
\hline & 3 & $20.42-27.67$ & 3.1145 & -0.0391 & 80007 & 0.0728 \\
\hline & 5 & $50.89-70.26$ & 2.8259 & -0.0462 & 80007 & 0.0641 \\
\hline
\end{tabular}

LOD Logarithm of odds, SRA Source of resistance allele, $R^{2}$ Explained phenotypic variation; "R1" and "R2" represent the field trial 2013-A and 2013-B and "Average" is the average value of 2013-A and 2013-B

markers D829-0 and D122-6 with a physical distance of $3.9 \mathrm{Mb}$ based on the B73 physical map (AGPv4).

In the winter of 2017 in Hainan, nine $F_{7}$ progeny (603 plants) were planted in Hainan, and five InDel markers (A1, A10, C2, C3 and C6) were designed to genotype new recombinants. Ten types of recombinants were identified and self-pollinated to produce $10 \mathrm{~F}_{8}$ families (1159 plants) that were planted in Taian in 2018. Ten markers (D550, D664, D829-0, A1, A10, C2, C3, C6, D122-6 and D1347) were used to genotype the 10 types of recombinants (I-X; Fig. 2b). Based on the statistically significant difference $(P<0.05)$ in DSI value between "80007" and "80044" homozygotes, recombinants b-I, b-III, and $\mathrm{b}-\mathrm{V}$ were considered to be resistant to MRDD (Fig. 2b), suggesting that $q M r d d 2$ is located in the heterozygous region of these recombinant types. In contrast, recombinant types b-II, b-IV, and b-VI - b-X did not show a statistically significant difference $(P>0.05)$ in DSI between "80007" and "80044" homozygotes (Fig. 2b), indicating that $q M r d d 2$ is in the homozygous region. Thus, $q M r d d 2$ was localized to the 2.81-Mb (AGPv4) (http://www.maize gdb.org) interval between markers A10 and C3 (Fig. 2b).

Seven types of recombinants were identified with markers A10 and C3 from the $\mathrm{F}_{8}$ progeny which were then self-pollinated to generate $7 \mathrm{~F}_{9}$ families (765 plants). All $\mathrm{F}_{9}$ progeny were planted in Hainan Province in the winter of 2018. Three markers (S33, D1116 and C2) in the mapped A10/C3 interval and another seven newly developed markers (D829-0, A1, A10, C3, C6, C13 and $\mathrm{C} 18$ ) were applied to genotype the 7 recombinants (Fig. 2c). The same progeny test strategy was utilized to determine whether there was a statistically significant difference $(P<0.05)$ in DSI value between "80007" and "80044" homozygotes. The results for type c-I, c-IV and $\mathrm{c}-\mathrm{VI}$ recombinants indicated that $q M r d d 2$ is located in the heterozygous region. Analogously, qMrdd2 was localized to the homozygous region of type c-II, c-III, c-V and
c-VII recombinants (Fig. 2c). Therefore, qMrdd2 was mapped between markers S33 and D1116 with a physical distance of 1.28-Mb (AGPv4).

We conducted the fourth fine-mapping in the summer of 2019 in Taian. Three InDel markers (N37, N31 and N42) were developed in the newly mapped 1.28-Mb region, and 1421 individuals from 9 recombinants were obtained from $\mathrm{F}_{9}$ progeny. The progeny of types d-I, d-IV, $\mathrm{d}-\mathrm{VI}$ and d-IX show significantly $(P<0.05)$ different DSI values, and types d-II, d-III, d-V, d-VII and d-VIII did not show significant differences $(P>0.05)$ in DSI. These data further allowed us to localize $q M r d d 2$ to the 577 kb (AGPv4) interval flanked by markers N31 and N42 (Fig. 2d).

\section{Genetic model of $q M r d d 2$ resistance to MRDD}

$\mathrm{F}_{6}, \mathrm{~F}_{8}$ and $\mathrm{F}_{9}$ families were used to investigate the genetic effect of $q M r d d 2$. Plants with homozygous "80007"/"80007", heterozygous "80007"/"80044" and homozygous "80044"/"80044" genotypes showed DSIs of $30.94,43.35$ and $51.48 \%$ in 2017 Taian, 30.94, 43.35 and $51.48 \%$ in 2018 Taian, 40.62, 52.28 and $66.23 \%$ in 2018 Hainan, respectively. For each field trial, homozygous "80044"/"80044" plants showed an obvious difference in DSI relative to homozygous "80007"/"80007" plants, with an average difference of $20.4 \%$. The DSI of heterozygous plants was, on average, $12.17 \%$ greater than that of homozygous "80007"/"80007" plants (Fig. 3). These results indicated that the $q M r d d 2$ QTL acts in an incompletely dominant manner to confer resistance to MRDD.

\section{Discussion}

The recombinant-derived progeny test is an efficient method and usually used to detect a QTL in backcrossed population such as $q R f g 2$ and qhmf4 [27-29]. Compared 


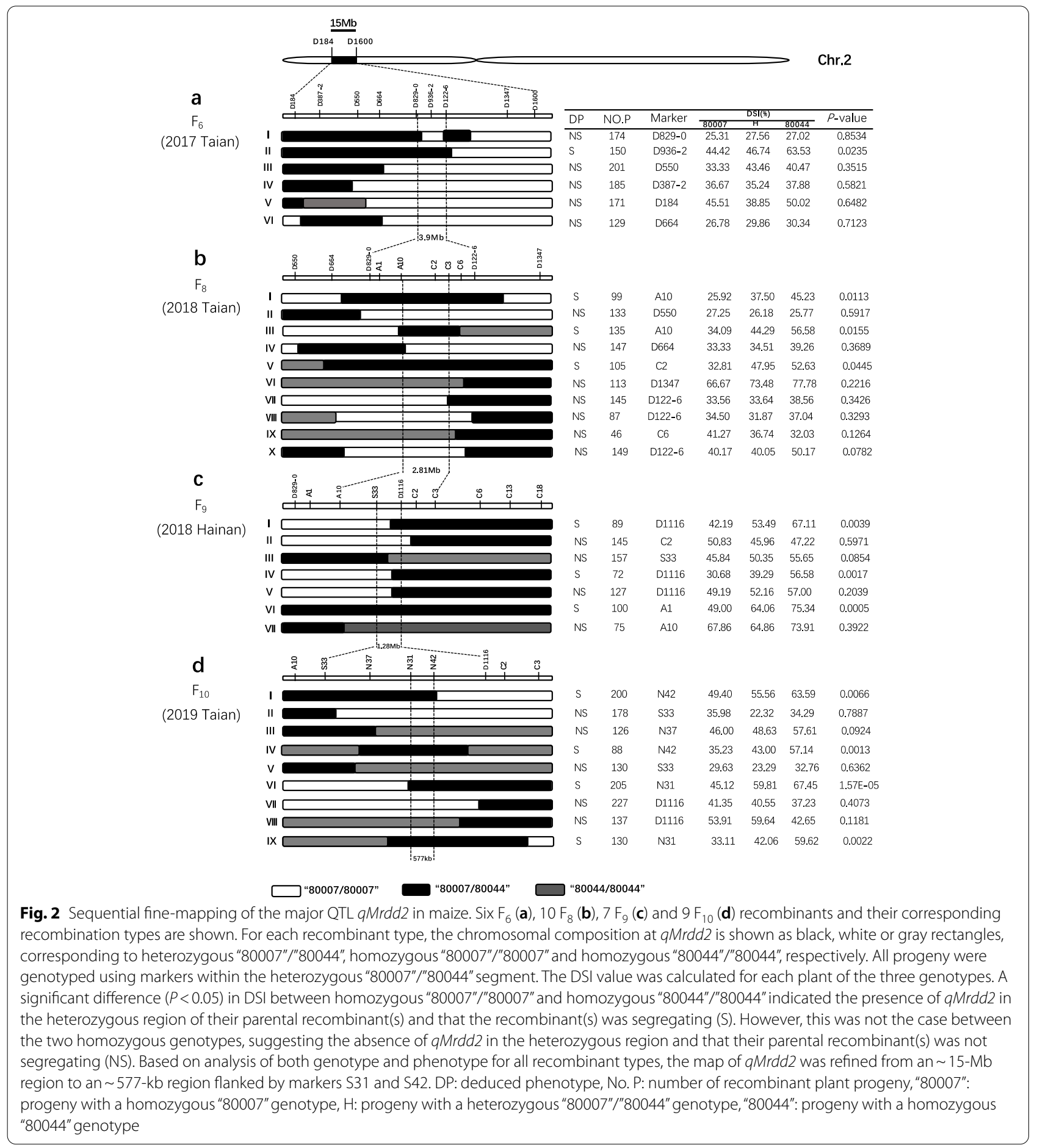

with backcrossed progeny, the self-pollinated progeny can capture the effect of all three genotypes and create more recombinants for fine- mapping. However, if recombinants are homozygous at both flanking markers, they produced by self-pollination cannot be used for fine-mapping. Crosses with other heterozygous plants in the same family can solve this problem very effective.
An artificial infection process [30] was used to evaluate the MRDD resistance of individual plants in this study, in which adequate viruliferous planthoppers carrying RBSDV provided stable and uniform disease pressure. In 2017 in Hainan, because the seedling age was inconsistent with the planthoppers, the planthoppers were old with low motility when the seed germinates. As a result, 


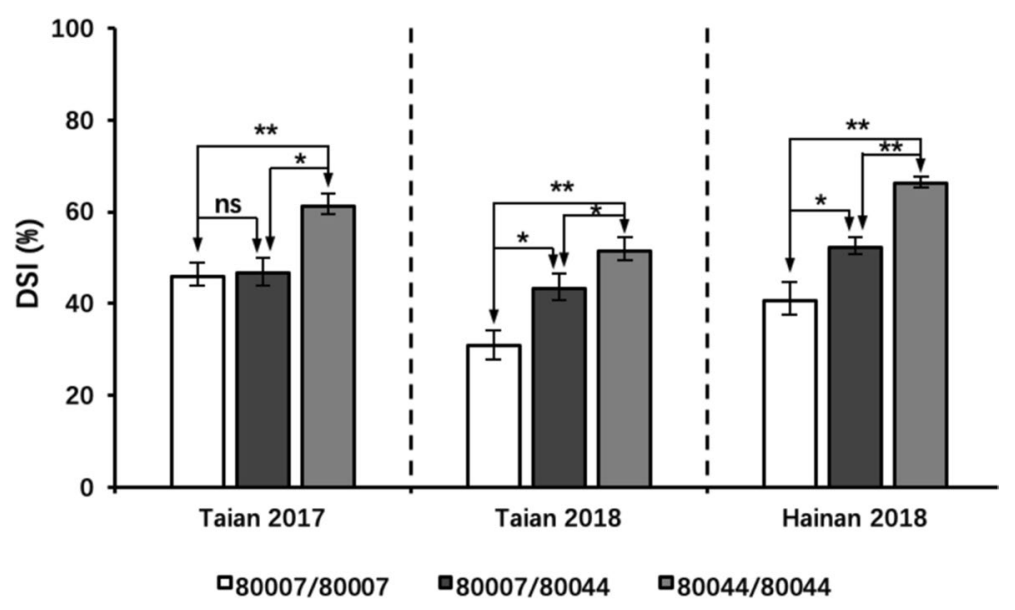

Fig. 3 Genetic effect of the QTL $q M r d d 2$ on $F_{n}$ populations across three experimental sites. $F_{n}$ populations were divided into three genotypes ("80007"/"80007", "80007"/"80044" and "80044"/" $80044 ")$ according to genotypes within the qMrdd2 region. Average DSI values are shown ( ${ }^{*} P<0.05$; **P $<0.01$; ns, not significant; two-tailed Student's t-test)

planthopper activity was inadequate to assess most of the recombinant individual phenotypes. In the spring of 2019 , disease-resistant phenotypes in the $\mathrm{F}_{9}$ population were not as robust as those in previous experiments, which may have resulted from a severe cold snap during the seedling stage. As the first survey of disease symptoms was conducted at 40 days after inoculation, the most severely affected plants could be analyzed before they died, followed by second and third surveys after pollination at 10-day intervals to ensure consistency of the phenotypic data.

We previously identified a major resistance QTL ( $q M r d d 1$ on chr.8) from the highly resistant inbred line NT411 and X178 [25]. Additional resistance QTLs have been identified on chr.1, 2, 5, 6, and 7 [19, 24, 31, 32]. In this study, using a different resistant inbred line (80007), we detected another major QTL ( $q M r d d 2$, chr.2, bin 2.01/02) and localized it to an interval of $\sim 577 \mathrm{~kb}$. A resistance QTL, previously mapped on chr.2 (bin 2.07/08) [8] is clearly different from $q M r d d 2$ (bin 2.01/02). Also, $q M r d d 2$ could be validated in the context of association mapping, by exploring a panel of accessions which usually contains a great diversity from one or multiple germplasms.

In this study, the $q M r d d 2$ was narrowed down to an $\sim 577 \mathrm{~kb}$ region (APV 4). Gene prediction by FEGNESH 2.6 (http://linux1.softberry.com/berry.phtml) has revealed 29 putative genes in the $q M r d d 2$ region. Most of the genes have not been annotated yet, only one ( $\mathrm{Zm00001d002312)}$ is related to disease resistance. The gene Zm00001d002312 was annotated as Leucinerich repeat transmembrane protein kinase (LRR-TM) in the MazieGDB and the LRR-TM genes are important receptor resistance genes in plants in response to pathogen infection [33]. Both $C f-2$ and HcrVf2 were this type of gene, which were related to the resistance to Cladosporium fulvum in tomato and Scab in apple [34, 35], respectively. While $Z m 00001 d 002312$ is a good candidate gene for $q M r d d 2$, further experiments such as genetic mapping by screening new recombinants within the $q M r d d 2$ locus or gene expression and transgenic testing studies must be performed to restrict the $q M r d d 2$ locus to a single gene.

Because $q M r d d 2$ is an incompletely dominant resistance QTL, the $q M r d d 2$ donor region should be integrated into both parental lines via MAS to guarantee maximally resistant $F_{1}$ hybrids. However, given that homozygous segments from both parental lines would impair heterosis, it is important to minimize the overlapped $q M r d d 2$ region in the $F_{1}$ hybrid for successful molecular breeding. We propose that one parental line carry the shortest possible $q M r d d 2$ donor region on the left, while the other parental line carry the shortest possible $q M r d d 2$ donor region on the right via MAS to minimize such overlap. Therefore, developing suitable markers to identify the shortest possible $q M r d d 2$ donor region to minimize such overlap is of great importance. In present study, the genetic distance between marker N31 and N42 was $\sim 0.67 \mathrm{cM}$. Thus, they may be effective for MAS of MRDD resistance because this marker is tightly linked within $5 \mathrm{cM}$ of the QTL [36].

\section{Conclusions}

It is critical to identify new resistance QTLs that can be used to genetically control MRDD and to deepen our understanding of resistance mechanisms. With 
the availability of a RIL population, we here detected a number of consistent MRDD QTL. Using a recombinant-derived progeny test strategy, A major resistance QTL ( $q M r d d 2)$ have been identified and successfully refined into $577 \mathrm{~kb}$ region. We further demonstrated that $q M r d d 2$ is an incompletely dominant resistance locus for MRDD that reduced the disease severity index by $20.4 \%$. This locus will be valuable for improving maize variety resistance to MRDD via MAS.

\section{Methods}

\section{Plant materials}

An RIL population consisting of 199 lines developed by single-seed descent from a cross between the inbred lines "80044" and "80007" was used in this study. The "80044" is a susceptible line bred from a Reid heterotic group, and " 80007 " is a resistant line from the P heterotic group. The two parents as well as the RILs were all developed by Prof. Baoshen Liu of Shandong Agricultural University. For the initial QTL mapping in 2013, we planted 199 RILs as the initial QTL mapping population for conducting two field trials (2013-A and 2013-B) at an experimental station (Jining, Shandong Province). For each RIL, 30 seeds were sown in one row with a spacing of $0.6 \mathrm{~m}$ between rows and $0.25 \mathrm{~m}$ between plants within a row. For fine mapping, 58 resistant RILs with different chromosomal recombinations in the QTL region were chosen and backcrossed with the susceptible line "80044" to produce $F_{1}$ populations. Using marker-assisted selection (MAS), the $F_{1}$ hybrids with the target QTL were self-pollinated four times to produce an $\mathrm{F}_{5}$ population; all mapping populations were developed based on the experimental flow chart presented in Fig. 4. All populations were grown at the experimental station of Shandong Agricultural University (Tai'an, Shandong Province) and experimental base (Sanya, Hainan Province) for fine mapping. For comparison, we planted two parents in each fine mapping, all populations and two parent lines were artificially inoculated with viruliferous planthoppers (see below).

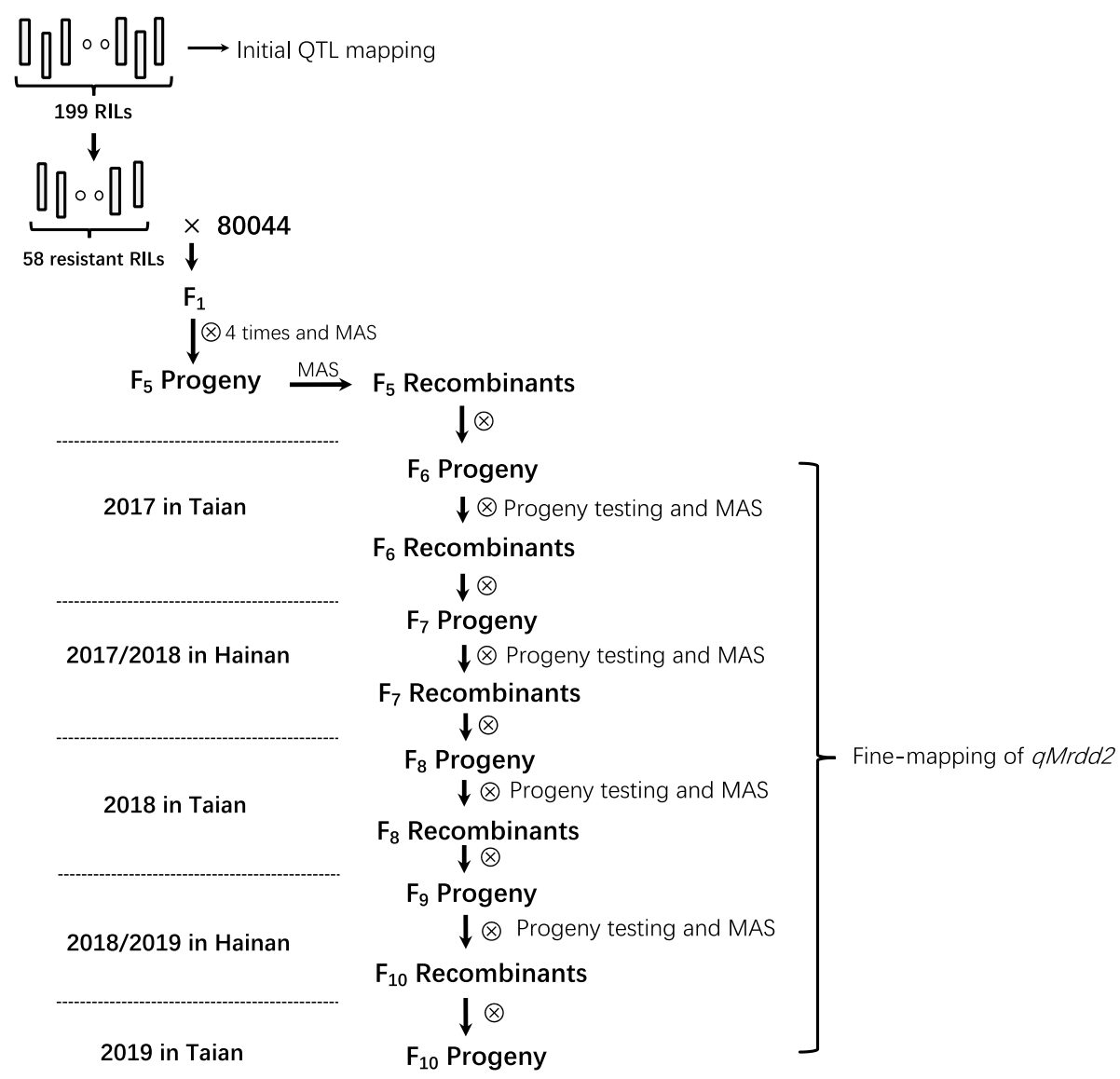

Fig. 4 Experimental flow chart for QTL identification and fine-mapping of QTL qMrdd2, which confers resistance to MRDD. A total of 199 RILs were used for the initial QTL mapping. Recombinants identified within the $F_{6}-F_{9}$ populations were genotyped and investigated to fine-map the QTL qMrdd2 
Molecular markers within the major QTL region were used to identify recombinants from the $F_{5}$ population, and the recombinants were self-pollinated to generate $F_{6}$ progeny. This process was repeated during 2017-2019 to develop a series of advanced self-pollinated populations consisting of $1010 \mathrm{~F}_{6}, 603 \mathrm{~F}_{7}, 1159 \mathrm{~F}_{8}, 765 \mathrm{~F}_{9}$ and 1421 $\mathrm{F}_{10}$ plants for fine mapping of $q M r d d 2$. MAS and progeny testing were conducted across self-pollinated populations until fine mapping of the target QTL was completed.

\section{Artificial inoculation and scoring of plants for symptoms}

Planthoppers carrying RBSDV were provided by Jiangsu Academy of Agricultural Sciences (Nanjing, China) [37]. Before inoculation, maize kernels derived from one recombinant plant were placed in a plastic casing, and a Dot-ELISA [38] was used to detect the infection rate of the planthoppers. Seedlings inoculated at the coleoptile stage with one infected planthopper [39] were cultured at $24{ }^{\circ} \mathrm{C}$ in a greenhouse for $72 \mathrm{~h}$; the seedlings were planted in a sample plot with $0.3-\mathrm{m}$ spacing between plants and 0.6-m spacing between rows.

Symptom scoring was conducted at 40 days after inoculation and was repeated twice after pollination at 10-day intervals. For QTL analysis and fine-mapping, a scoring system $(0,0.25,0.5,0.75$ or 1$)$ based on overall symptoms was adopted to evaluate MRDD resistance (Fig. 5), as follows: $0=$ no symptoms; $0.25=$ slightly shortened superior internodes, resulting in plants that are $\sim 80 \%$ of the height of a healthy plant; $0.5=$ dark-green leaves, waxy enations on abaxial surfaces of leaves and sheaths and obviously shortened superior internodes, resulting in plants that are $\sim 66 \%$ of the height of a healthy plant; $0.75=$ severely shortened internodes and malformed tassels, resulting in plants that are $\sim 50 \%$ of the height of a healthy plant; $1=$ severe stunting, with suppressed flowering and the absence of ears, resulting in plants that are $<50 \%$ of the height of a healthy plant. DSI was calculated by the following formula: DSI $(\%)=\sum[$ (scale $\times$ number of plants in scale $) /(1 \times$ total number of plants $)] \times 100 \%$, and was used to represent the MRDD severity of families [40].

\section{Analysis of phenotypic data}

Statistical analysis was performed using $\mathrm{R}$ project [39]. Two sets of phenotypic data (2013-A, 2013-B) were collected for the RIL population and the Spearman's rank correlation coefficient $\left(r_{s}\right)$ of them was calculated. We used ANOVA (part of the R project) to test the significance of the genotypic and environmental effects. The model for variance analysis is as follows: $y_{i}=\mu+g i+e j+\varepsilon$, in which $y_{i}$ is the phenotypic value of the $i$ th RIL line, $\mu$ is the overall average value, $g i$ the genetic effect of $i$ th RIL, $e j$ the environmental effect of $j$ th trial and $\varepsilon$ is the residual. The ANOVA result was used to calculate the broad-sense heritability $\left(H^{2}=\sigma_{g}^{2} /\right.$ $\left.\sigma_{g}^{2}+\sigma_{\varepsilon}^{2} / e\right)$, where $\sigma_{g}^{2}$ is the genetic variance, $\sigma_{\varepsilon}^{2}$ is the residual error, and $e$ is the number of environments $[41,42]$.

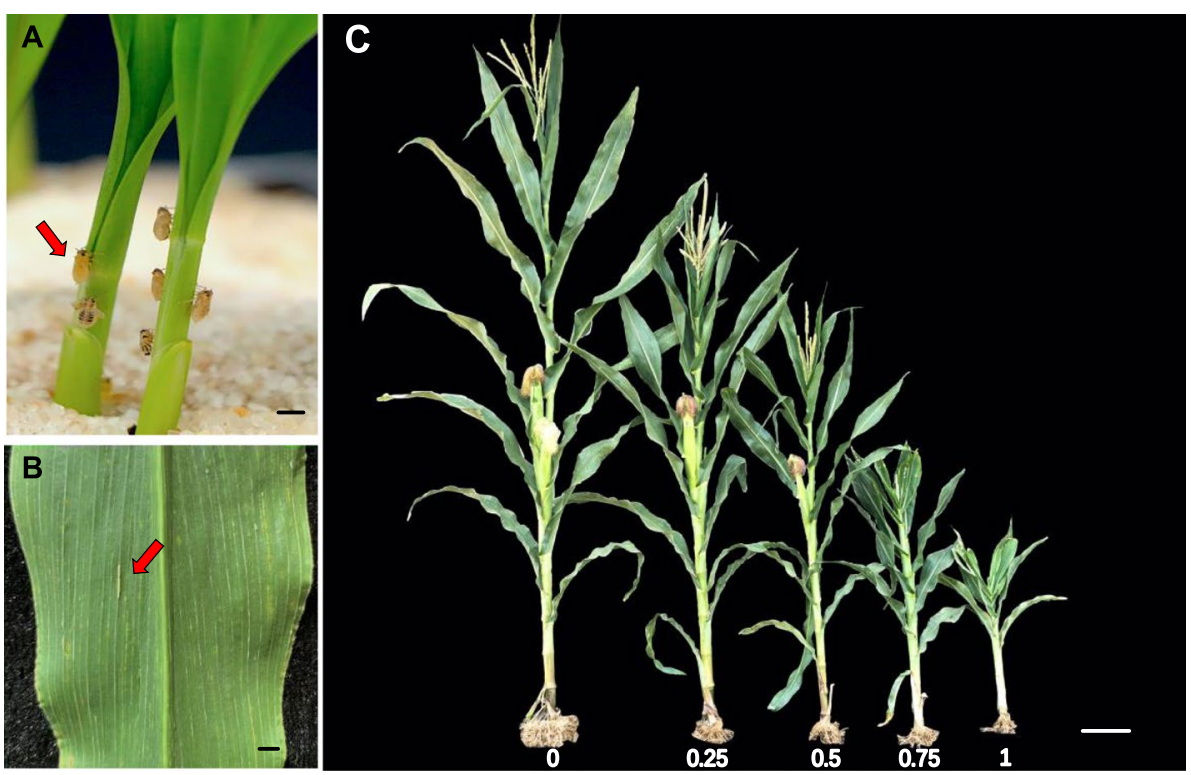

Fig. 5 Symptom of RBSDV on Zea mays L. A Rice black-streaked dwarf virus (RBSDV) is transmitted to its natural host, maize, by the brown planthopper (Laodelphax striatellus). Bar $=5 \mathrm{~mm}$. B Waxy enations on abaxial surfaces of leaves present in maize plants infected with RBSDV. Bar $=5 \mathrm{~mm}$. C Maize with MRDD scores of 0-1. Disease scores were primarily based on plant height. Bar $=10 \mathrm{~cm}$ 
Student's t-test was performed in QTL fine-mapping study to compare the difference in DSI between the two parent lines "80007" and "80044" and the three genotypes ("80007"|"80007", "80007"/"80044" and "80044"|"80044") in self-pollinated populations.

\section{Linkage map construction and detection of QTLs for resistance to MRDD}

Genotype data for the RIL population were collected using an Illumina Golden Gate $3 \mathrm{~K}$ SNP chip. We obtained 3072 SNP markers from Illumina Golden Gate 3 K SNP chip and six criteria were applied to analysis SNP database (Table S5). Based on these genotype data, we employed JoinMap (Version 2.5) [43] to construct a genetic linkage map and the Kosambi mapping function [44] to calculate the genetic distances. QTL analysis was carried out in Windows QTL Cartographer (Version 2.5) [45] with the composite interval mapping method [46], a walk speed of $2.0 \mathrm{cM}$ and a window size of $10 \mathrm{cM}$. For each of the datasets (R1, R2, and Average), a significance threshold to confirm a putative QTL was obtained from a 1000 -permutation test at $\mathrm{P}<0.05$ with a logarithm of the odds (LOD) score $>2.5$ [47].

\section{Genotyping}

Leaf tissue from each plant in the field was collected at the six- to seven-leaf stage. An SDS procedure [48] was used to extract genomic DNA. Maize insertion or deletion (InDel) and SSR markers were retrieved from MaizeGDB (http://www.maizegdb.org/); the primers were produced by Sangon Biotech (Shanghai, China). Each sample was subjected to genotyping using the markers as required, and PCR products were separated by $1 \%$ agarose gel or $6 \%$ polyacrylamide gel electrophoresis.

\section{Development of PCR-based markers}

Based on the initial QTL analysis, $q M r d d 2$ was mapped to the confidence region between D184 and D1600, which covers a physical distance of $15 \mathrm{Mb}$ based on the B73 physical map (AGPv4). Therefore, we saturated the region with high-density molecular markers. Two types of markers were developed based on different sequence variations, including SSRs and InDels. To develop SSR markers, the maize B73 reference sequence in the QTL region was downloaded from the website http://plants. ensembl.org/Zea_mays/Info/Index/. The sequences were scanned by the software SSRHunter (Version 1.3) to search for SSRs, and primers flanking the SSRs were designed with the PrimerQuest tool (https://sg.idtdna. com/Primerquest/Home/Index). To develop InDel markers, lines "80007" and "80044" were sequenced by Tsingke Biotech (Beijing, China), and InDels in the $15 \mathrm{Mb}$ interval for the QTL were analyzed. Single-/low-copy sequences were detected by BLAST on MaizeGDB with the B73 genomic sequence database. BLAST results were surveyed for InDels of $\geq 3 \mathrm{bp}$, which were detected as 3 -bp gaps in the alignment of sequences from the two parents. InDels with $\geq 1000$ bp of upstream sequence and $\geq 1000 \mathrm{bp}$ of downstream sequence were assessed by PCR. Primers were designed by Primers Input (https:// primer3.ut.ee) with the targets $(970,60)$. All primers met to the following criteria: $\sim 20$ nucleotides with $40-60 \%$ GC content, no consecutive tracts of a single nucleotide and no secondary structure. Ultimately, 21 markers (10 SSRs and 11 InDels) were found to be polymorphic between the parental lines (Table S4).

\section{Strategy for fine-mapping of $q M r d d 2$}

Appropriate marker density, crossover density and the ability to determine the precise phenotype of each recombinant are essential for fine mapping analysis. Based on the initial QTL mapping, we developed highdensity markers within the $15-\mathrm{Mb}$ interval of $q M r d d 2$. Sequential fine mapping of $q M r d d 2$ was carried out by using recombinant-derived progeny [27]. In this study, a robust progeny test strategy was employed to evaluate the MRDD phenotype of all recombinants (Fig. 4). The DSI was used to represent MRDD severity, as previously described in detail (Fig. 5). Recombinants developed from the $F_{n}$ population were self-pollinated, and the resulting self-pollinated population was grown in the same experimental stations under artificial infection. Each individual was genotyped using markers in the heterozygous region of its parental recombinant and scored for disease severity in the field. A DSI value was estimated for each resulting self-pollinated population. The progeny from each recombinant strain were divided into three genotypes: "80007" homozygote, "80044" homozygote and "80007"/"80044" heterozygote. Statistically significant differences in the DSI value of each genotype were determined using two-tailed Student's $t$-test. If there was a significant difference $(P<0.05)$ in DSI value between the two homozygous genotypes, $q M r d d 2$ was considered to be located in the ' 80007 ' donor segment, namely, in the heterozygous region of the parental recombinant. If there was no significant difference $(P \geq 0.05)$ in DSI value between the two homozygous genotypes, $q M r d d 2$ was assumed to be absent in the '80007' donor segment, namely, in the homozygous region of the parental recombinant, or no $q M r d d 2$ was present.

\section{Abbreviations}

MRDD: Maize rough dwarf disease; QTL: Quantitative trait loci; SSR: Simple sequence repeat; RIL: Recombinant inbred line; MAS: Marker-assisted selection; MRDV: Maize rough dwarf virus; MRCV: Mal de Rio Cuarto virus; SRBSDV: Southern rice black-streaked dwarf virus; InDel: Insertion or deletion. 


\section{Supplementary Information}

The online version contains supplementary material available at https://doi. org/10.1186/s12870-021-03107-1.

Additional file 1: Table S1. Spearman's rank correlation coefficient $\left(r_{s}\right)$ of DSI between the two field trials. Table S2. Marker and genetic distance information for the 10 maize linkage groups. Fig. S1. The ten maize genetic linkage groups. Fig. S2. Linkage disequilibrium (LD) heat map of each linkage group. Table S3. The number of SNP markers accepted as cofactors in the statistical model of QTL mapping. Fig. S3. Detection of QTLs conferring resistance to MRDD. Fig. S4. Boxplot graph of DSI values of the initial mapping population. Table $\mathbf{S 4}$. Markers developed to map the qMrdd2 locus. Table S5. Criteria applied to analysis SNP database.

\section{Acknowledgements}

Not Applicable.

\section{Authors' contributions}

WXZ performed most of the experiments and wrote the paper. SND and QCL performed the initial QTL mapping, maize cultivation and pollination. YZ performed the artificial inoculation, phenotypic evaluation, and pollination. WX performed the artificial inoculation, pollination, and genotyping. YZZ participated in the phenotypic evaluation and pollination. MLX guided the experiments and was also involved in the analysis of the results. CMR and $\mathrm{ZBC}$ provided the brown planthopper. BSL designed and supervised the experiments and provided the RIL population. BSL edited and revised the manuscript. All authors read and approved the final manuscript.

\section{Funding}

This work was jointly funded by the National Key Research and Development Program of China (2016YFD0101803 to BL), the National Natural Science Foundation of China (31973950 to BL), and Shandong Improved Variety Program (2019LZGC002 and 2017LZGC005 to BL).

\section{Availability of data and materials}

The sequencing data is available from the corresponding author on reasonable request. Other data produced during this study are included in our article and in its supplementary files.

\section{Declarations}

\section{Ethics approval and consent to participate}

Not applicable.

\section{Consent for publication}

Not applicable.

\section{Competing interests}

The authors declare that they have no competing interests.

\section{Author details}

'State Key Laboratory of Crop Biology, Shandong Agricultural University, 61 Daizong Street, Taian 271018, People's Republic of China. ${ }^{2}$ National Maize Improvement Center, China Agricultural University, 2 West Yuanmingyuan Road, Beijing 100193, People's Republic of China. ${ }^{3}$ Institute of Plant Protection, Jiangsu Academy of Agricultural Sciences, Nanjing 210014, People's Republic of China.

Received: 14 March 2021 Accepted: 16 June 2021

Published online: 30 June 2021

\section{References}

1. Xie L, LV MF, Yang J, Chen JP, Zhang HM. Genomic and phylogenetic. evidence that Maize rough dwarf and rice black-streaked dwarf fijiviruses should be classified as different geographic strains of a single species. Acta Virol. 2017:61:453-62.
2. Dovas Cl, Eythymiou K, Katis NI. First report of maize rough dwarf virus (MRDV) on maize crops in Greece. Plant Pathol. 2004;53:238.

3. Arneodo JD, Guzman FA, Conci LR, Laguna IG, Truol GA. Transmission features of Mal de Río Cuarto virus in wheat by its planthopper vector Delphacodes kuscheli (Hemiptera: Delphacidae). Ann Appl Biol. 2002;141:195-200.

4. Bai F, Yan J, Qu Z, Zhang H, Xu J, Ye M, Shen D. Phylogenetic. Analysis reveals that a dwarfing disease on different cereal crops in China is due to Rice Black Streaked Dwarf Virus (RBSDV). Virus Genes. 2002;25:201-6.

5. Xiao Y, Xu F, Zheng F, Li X, Liu B, Zhang C. Molecular. Characterization of segments S7 to S10 of a Southern Rice Black-streaked Dwarf Virus isolate from maize in northern China. Virol Sin. 2011;26:47.

6. Caciagli P, Casetta A. Maize rough dwarf virus (Reoviridae) in its. planthopper vector Laodelphax striatellus in relation to vector infectivity. Ann Appl Biol. 2010;109:337-44

7. Milne RG, Lovisolo O. Maize rough dwarf and related viruses. Adv Virus Res. 1977;21:267-341.

8. Shi L, Hao Z, Weng J, Xie C, Liu C, Zhang D, Li M, Bai L, Li X, Zhang S. Identification of a major quantitative trait locus for resistance to maize rough dwarf virus in a Chinese maize inbred line X178 using a linkage map based on 514 gene-derived single nucleotide polymorphisms. Mol Breeding. 2011:30:615-25.

9. Zhang H, Chen J, Lei J, Adams MJ. Sequence analysis shows that a dwarfing disease on rice, wheat and maize in China is caused by Rice Black-streaked Dwarf Virus. Eur J Plant Pathol. 2001;107:563-7.

10. Tao Y, Liu Q, Xu M. Research progress on maize rough dwarf. Dis J Maize Sci. 2013;21(1):149-52.

11. Bartsur A. Resistance of corn genotypes to maize rough dwarfs virus [in Israel]. Maydica. 1988;33:189-200.

12. Wang AL, De Fa Z, Chen ZH. Studies on genetic basis and recurrent. selection effect of inbred line maize resistance to MRDV. Maizeences. 2000;8(1):80-2.

13. Guo QL. The observation and analysis of varietal resistance of maize. Rough Dwarf Virus Dis Plant Prot. 1995;1:21-3.

14. Shang Y, Zhao J, Du S, Lu X, Wang S, Sun H, Yang C. Identification and investigation on resistance to virus diseases of both maize commercial varieties and germplasm at seedling stage. Shandong Agric Sci. 2001:4:3-5

15. Wang G, Han H, Zhao F, Wang H, Kong X, Ye J. Identification on disease resistance of maize varieties (lines) to maize rough dwarf virus. Acta Agric Zhejiangensis. 2011;23:0-567.

16. Xue L, Zhang D, Liang $X$, Jin $M$, Peng C, Xu C. Mining and analyzing genetic diversity for maize rough dwarf disease re-sistant gerplasms and its application in maize breeding. Acta Agron Sin. 2011;37:2123-9.

17. Di Renzo MA, Bonamico NC, Díaz DD, Salerno JC, Ibañez MM, Gesumaria JJ. Inheritance of resistance to Mal de Rio Cuarto (MRC) disease in Zea mays (L.). J Agric Sci. 2002;139:47-53.

18. Bonamico NC, Diaz DG, Ibanez MA, Faricelli ME, Balzarini MG, Salerno JC. Microsatellite markers linked to QTL for resistance to Mal de Río Cuarto disease in Zea mays L. J Agri Sci. 2004;142:289-95.

19. Luan JW, Wang F, Li YJ, Zhang B, Zhang JR. Mapping quantitative trait loci conferring resistance to rice black-streaked virus in maize (Zea mays L.). Theor Appl Genet. 2012;125(4):781-91.

20. Shi L, Weng J, Liu C, Song X, Miao H, Hao Z, Xie C, Li M, Zhang D, Bai L, Pan G, Li X, Zhang S. Identification of promoter motifs regulating ZmelF4E expression level involved in maize rough dwarf disease resistance in maize (Zea Mays L.). Mol Genetics Genomics. 2013;288(3-4):89-99.

21. Shi LY, Hao ZF, Weng JF, Xie CX, Liu CL, Zhang DG, Li MS, Bai L, Li XH, Zhang $\mathrm{SH}$. Identification of a major quantitative trait locus for resistance to maize rough dwarf virus in a Chinese maize inbred line X178 using a linkage map based on 514 gene-derived single nucleotide polymorphisms. Mol Breeding. 2011;30(2):615-25.

22. Liu C, Hua J, Liu C, Zhang D, Hao Z, Yong H, Xie C, Li M, Zhang S, Weng J. Fine mapping of a quantitative trait locus conferring resistance to maize rough dwarf disease. Theor Appl Genet. 2016;129:2333-42.

23. Chen G, Wang X, Hao J, Yan J, Ding J. Genome-wide association implicates candidate genes conferring resistance to maize rough dwarf disease in maize. Plos One. 2015;10(11):e0142001.

24. Li R, Wei S, Wang B, Wang J, Zhang D, Zhang Q, Li X, Wei J, Gao Z. Identification of a locus conferring dominant resistance to maize rough dwarf disease in maize. Sci Rep. 2018;8:3248. 
25. Tao Y, Liu Q, Wang H, Zhang Y, Huang X, Wang B, Lai J, Ye J, Liu B, Xu M. Identification and fine-mapping of a QTL, qMrdd1, that confers recessive resistance to maize rough dwarf disease. BMC Plant Biol. 2013;13:145.

26. Liu Q, Deng S, Liu B, Tao Y, Ai H, Liu J, Zhang Y, Zhao Y, Xu M. A helitroninduced RabGDla variant causes quantitative recessive resistance to maize rough dwarf disease. Nat Commun. 2020;2020:495.

27. Yang $Q$, Zhang D, Xu M. A sequential quantitative trait locus fine-mapping strategy using recombinant-derived progeny. J Integr Plant Biol. 2012;54:228-37.

28. Ye J, Zhong T, Zhang D, Ma C, Wang L, Yao L, Zhang Q, Zhu M, Xu $M$. The auxin-regulated protein ZmAuxRP1 coordinates the balance between root growth and stalk rot disease resistance in maize. Mol Plant. 2019;012(003):360-73.

29. Ren J, Wu P, Tian X, Thomas L, Chen S. QTL mapping for haploid male fertility by a segregation distortion method and fine mapping of a key QTL ahmf4 in maize. Theor Appl Genet. 2017;130(7):1-11.

30. Ren C, Yang L, Liu B, Yuan J, Miao Q, Lu F, Cheng Z. Establishment and application for a high throughput artificial inoculation method of rice black-streaked dwarf virus to maize. Mazie Sci. 2018;26:142-8.

31. Bonamico NC, Balzarini MG, Arroyo AT, Ibañez MA, Dĺaz DG, Salerno JC, Di Renzo MA. Association between microsatellites and resistance to Mal de Río Cuarto in maize by discriminant analysis. Phyton. 2010;79:31-8.

32. Di Renzo MA, Bonamico NC, DÍaz DG, Ibañez MA, Faricelli ME, Balzarin MG, Salerno JC. Microsatellite markers linked to QTL for resistance to Mal de Río Cuarto disease in Zea mays L. J Agric Sci. 2004;142(3):289-95.

33. Martin CB, Bogdanove AJ, Sessa G. Understanding the functions of plant disease resistance proteins. Annu Rev Plant Biol. 2003:54:23-61.

34. Krüger J, Thomas CM, Golstein C, Dixon MS, Smoker M, Tang S, et al. A tomato cysteine protease required for $\mathrm{Cf}$-2-dependent disease resistance and suppression of autonecrosis. Science. 2002;296:744-7.

35. Vinatzer BA, Patocchi A, Gianfranceschi L, Tartarni S, Zhang HB, Gessler C, Sansavini S. Apple contains receptor-like genes homologous to the Cladosporium fulvum resistance gene family of tomato with a cluster of genes cosegregating with $\mathrm{Vf}$ apple scab resistance. Mol Plant Microbe Interact. 2001;14(4):508-15.
36. Michelmore R. Molecular approaches to manipulation of disease. resistance genes. Annu Rev Phytopathol. 1995;33:393-427.

37. Ren C, Cheng Z, Yang L, Miao Q, Fan Y, Zhou Y. A novel, in vivo, indoor method to preserve rice black-streaked dwarf virus in small brown planthopper using wheat seedling as a bridge host. J Virol Methods. 2014;208:26-32.

38. Zhou Y, Liu H, Wang G, Huang X, Cheng Z, Chen Z, Zhou X. Immunity detecting of rice stripe virus in Laodelphax striatellus. Jiangsu Agric Sci. 2004;1:50-1.

39. The R project for statistical computing. https://www.r-project.org/.

40. Grau CR. Resistance of soybean cultivars to Sclerotinia sclerotiorum. Plant Dis. 1982;66:506-8.

41. Holland JB. Estimating and interpreting heritability for plant breeding: an update. Plant Breed Rev. 2003:22:9-112.

42. Kaapp SJ, Stroup WW, Ross WM. Exact confidence intervals for heritability on a progeny mean basis. Crop Sci. 1985. https://doi.org/10.2135/crops ci1985.0011183X002500010046x.

43. Van Ooijen JW. JoinMap 4 Software for the calculation of genetic linkage maps in experimental populations. Wageningen: Kyazma B V; 2006.

44. Kosambi $D$. The estimation of map distance from recombination values. Ann Eugen. 1944;12(1):172-5.

45. Basten CJ, Weir BS, Zeng ZB. QTL cartographer: a reference manual and tutorial for QTL mapping. Raleigh: Department of Statistics, North Carolina State University; 1997

46. Zeng Z. Precision mapping of quantitative trait loci. Genetics. 1994;136(4):1457-68.

47. Doerge RW, Churchill GA. Permutation tests for multiple loci affecting. a quantitative character. Genetics. 1996;142:285-94.

48. Murray MG, Thompson WF. Rapid isolation of high molecular weight plant DNA. Nucleic Acids Res. 1980;8:4321-6.

\section{Publisher's Note}

Springer Nature remains neutral with regard to jurisdictional claims in published maps and institutional affiliations.
Ready to submit your research? Choose BMC and benefit from:

- fast, convenient online submission

- thorough peer review by experienced researchers in your field

- rapid publication on acceptance

- support for research data, including large and complex data types

- gold Open Access which fosters wider collaboration and increased citations

- maximum visibility for your research: over 100M website views per year

At $\mathrm{BMC}$, research is always in progress.

Learn more biomedcentral.com/submissions 